\title{
Dephasing in (Ga,Mn)As nanowires and rings
}

\author{
K. Wagner* D. Neumaier, M. Reinwald, W. Wegscheider, and D. Weiss \\ Institut für Experimentelle und Angewandte Physik, Universität Regensburg, Regensburg, Germany
}

(Dated: March 9, 2018)

\begin{abstract}
To understand quantum mechanical transport in ferromagnetic semiconductor the knowledge of basic material properties like phase coherence length and corresponding dephasing mechanism are indispensable ingredients. The lack of observable quantum phenomena prevented experimental access to these quantities so far. Here we report about the observations of universal conductance fluctuations in ferromagnetic $(\mathrm{Ga}, \mathrm{Mn})$ As. The analysis of the length and temperature dependence of the fluctuations reveals a $T^{-1}$ dependence of the dephasing time.
\end{abstract}

PACS numbers: 75.50.Pp, 73.63.-b, 72.20.My

The discovery of the ferromagnetic III-V semiconductor materials (In,Mn)As [1] and (Ga,Mn)As [2] has generated a lot of interest as these materials combine ferromagnetic properties, typical for metals, with the versatility of semiconductors (for a review see e.g. [3, 4, 5, 6, 7]). This allows, e.g., to control ferromagnetism by electric fields thus opening new prospects for application and fundamental research [8]. The $\mathrm{Mn}$ atoms in the III-V host are not only responsible for the ferromagnetism but also act as acceptors such that, at sufficiently high Mn-concentration (Ga,Mn)As is a degenerate p-type semiconductor [9]. The ferromagnetic order of the Mn magnetic moments is mediated by holes via the Ruderman-Kittel-Kasuya-Yosida (RKKY) interaction [10]. The quest to increase the Curie temperature $T_{\mathrm{C}}$ in $(\mathrm{Ga}, \mathrm{Mn})$ As towards room temperature has led to a thorough investigation of the material properties (see, e.g. 11] and references therein). By annealing ( $\mathrm{Ga}, \mathrm{Mn})$ As sheets or by incorporating them into sophisticated layered arrangements the Curie temperature was increased up to $173 \mathrm{~K}$ [12, 13] and $250 \mathrm{~K}$ with $\mathrm{Mn} \delta$ doping [14], respectively. Despite the high crystalline quality of the material $(\mathrm{Ga}, \mathrm{Mn}) \mathrm{As}$ is a quite disordered conductor on the verge of the metal-insulator transition (MIT). For Mn concentrations on the metallic side of the MIT the typical mean free path of the holes are a few lattice constants. Hence quantum effects like Shubnikov-de Haas or Aharonov-Bohm (AB) oscillations or universal conductance fluctuations (UCF) were not yet reported. Hence the phase coherence length $L_{\phi}$ and the corresponding dephasing mechanisms which govern quantum mechanical interference phenomena in ferromagnetic semiconductors are not known yet. Below we report the observation of UCF in nanoscale $(\mathrm{Ga}, \mathrm{Mn})$ As wires at very low temperatures and probe currents. From an analysis of the temperature and length dependence of the UCF's information about phase coherence and dephasing in $(\mathrm{Ga}, \mathrm{Mn}) \mathrm{As}$ is obtained.

For our experiments, nanoscale wire and ring samples were fabricated from two different wafers grown by low

*Electronic address: konrad.wagner@physik.uni-regensburg.de

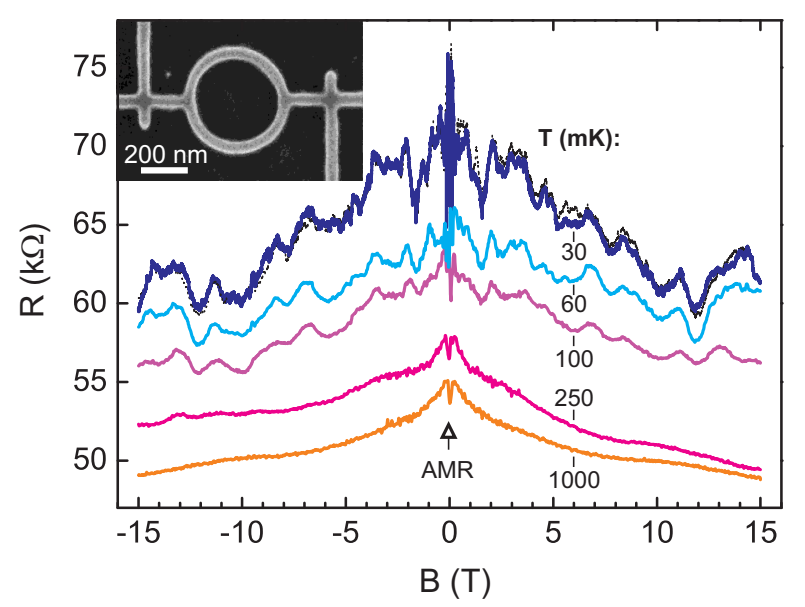

FIG. 1: (color online). Magnetoresistance of a (Ga,Mn)Asring with a diameter of $400 \mathrm{~nm}$ and a ring width of $40 \mathrm{~nm}$. The inset displays a top view of the ring. The temperature was varied from $1 \mathrm{~K}$ (bottom trace) to $30 \mathrm{mK}$ (top trace) and the current through the device was set to $100 \mathrm{pA}$. To demonstrate the reproducibility of the resistance oscillations observed below $\sim 200 \mathrm{mK}$ the $30 \mathrm{mK}$ trace is shown for an up-(blue line) and down-sweep (dashed black line) of the $B$ field.

temperature molecular beam epitaxy on semi insulating GaAs (100) substrates 15. The (Ga,Mn)As layers were $50 \mathrm{~nm}$ thick with a Mn concentration of $\sim 2 \%$. From transport experiments $T_{\mathrm{C}}$ was evaluated to be $\sim 60 \mathrm{~K}$. The sheet resistance $R_{\square}$ is $1.8 \mathrm{k} \Omega$ at $1 \mathrm{~K}$ corresponding to a dimensionless conductance $g=\frac{1}{R_{\square}}\left(\frac{e^{2}}{h}\right)^{-1} \approx 14 \gg 1$. The hole carrier concentration $p$, extracted from the highfield Hall resistance $R_{x y}$ at $90 \mathrm{mK}$ is $\sim 1.8 \times 10^{20} \mathrm{~cm}^{-3}$ [29]. Within a 6 band $k \cdot p$ model this corresponds to a Fermi energy of $130 \mathrm{meV}$ [16]. Using the joint effective mass of $0.61 m_{0}$ for heavy and light hole bands in GaAs we obtain a momentum relaxation time $\tau_{p}=1.3 \mathrm{fs}$. Here, $m_{0}$ is the free electron mass. Thus the low temperature diffusion constant $D=\frac{1}{3} v_{F}^{2} \tau_{p}=4.8 \times 10^{-5} \mathrm{~m}^{2} / \mathrm{s}$ where $v_{F}$ is the average Fermi velocity of heavy and light holes.

For transport experiments (Ga,Mn)As layers were first pre-patterned with a Hall-bar structure using standard 
optical lithography and chemical dry etching. Au contacts were made by standard lift-off method after brief insitu ion beam etching of the surface to remove the native oxide layer. To define wires and rings we used electron beam lithography (a LEO Supra 35 SEM and nanonic pattern generator) and reactive ion etching. Wires with lengths of $100 \mathrm{~nm}$ to $15 \mu \mathrm{m}$ and a nominal width as small as $20 \mathrm{~nm}$ (see inset of Fig. 2) were fabricated. To probe the $\mathrm{AB}$ effect we also fabricated rings with diameters of $100 \mathrm{~nm}$ to $800 \mathrm{~nm}$ and widths of $20 \mathrm{~nm}$ to $40 \mathrm{~nm}$. An example of a ring with $400 \mathrm{~nm}$ diameter is shown as inset in Fig. 1 The magnetotransport properties were measured in a top-loading dilution refrigerator with a base temperature of $15 \mathrm{mK}$ and magnetic fields up to $19 \mathrm{~T}$ (Oxford Kelvinox TLM Dilution Refrigerator with Femtopower Thermometry using $\mathrm{RuO}_{2}$ sensors calibrated down to $20 \mathrm{mK}$ ). To avoid heating we used a low frequency $(17 \mathrm{~Hz})$ and low current $(10 \mathrm{pA}$ to $100 \mathrm{pA})$ four probe lock-in technique. Low noise preamplifiers and careful wiring and filtering were crucial to observe quantum interference effects in this material.

In an attempt to observe $\mathrm{AB}$ oscillation we fabricated ( $\mathrm{Ga}, \mathrm{Mn}) \mathrm{As}$ rings and measured their resistance as a function of a perpendicular magnetic field $B$ and temperature $T$. Corresponding data are shown in Fig. 1. At high temperatures of $1 \mathrm{~K}$ and $250 \mathrm{mK}$ the magnetoresistance exhibits the behavior characteristic of $(\mathrm{Ga}, \mathrm{Mn}) \mathrm{As}$ layers: The resistance first increases up to a field of $\sim 120 \mathrm{mT}$ and then decreases over the entire investigated field range. The low-field increase is ascribed to the anisotropic magnetoresistance effect (AMR) 17] which flattens once the magnetization is saturated. The negative magnetoresistance is commonly assigned to decreasing magnetic disorder [18] but by others also seen as a signature of weak localization [19]. At temperatures below $\sim 200 \mathrm{mK}$ reproducible resistance fluctuations emerge but no $\mathrm{AB}$ oscillations are seen. The latter is expected if the ring circumference exceeds the phase coherence length $L_{\phi}$ significantly. The amplitude of the resistance fluctuations depends strongly on temperature and current. Hence the current through the ring was limited to $100 \mathrm{pA}$ here. The resistance fluctuations are especially pronounced in the low field interval dominated by the AMR. These fluctuations are out of the scope of the discussion below. As the magnetization in this low field regime is expected to be inhomogeneous an analysis of these fluctuations is complicated and might require a detailed knowledge of the local magnetization. Hence we focus on the high field fluctuations which occur in a $B$-regime of homogeneous magnetization below. Such a reproducible (within one cooling cycle) stochastic magnetoresistance pattern for a specific sample, also denoted as 'magnetofingerprints', is known as UCF [20].

To investigate the fluctuations we measured the conductance of (Ga,Mn)As wires with lengths between $100 \mathrm{~nm}$ and $15 \mu \mathrm{m}$ and a width of $20 \mathrm{~nm}$. Since the Hall resistance contribution $R_{x y}$ stemming from both anomalous and regular Hall effect is by a factor of $\sim 20$ smaller

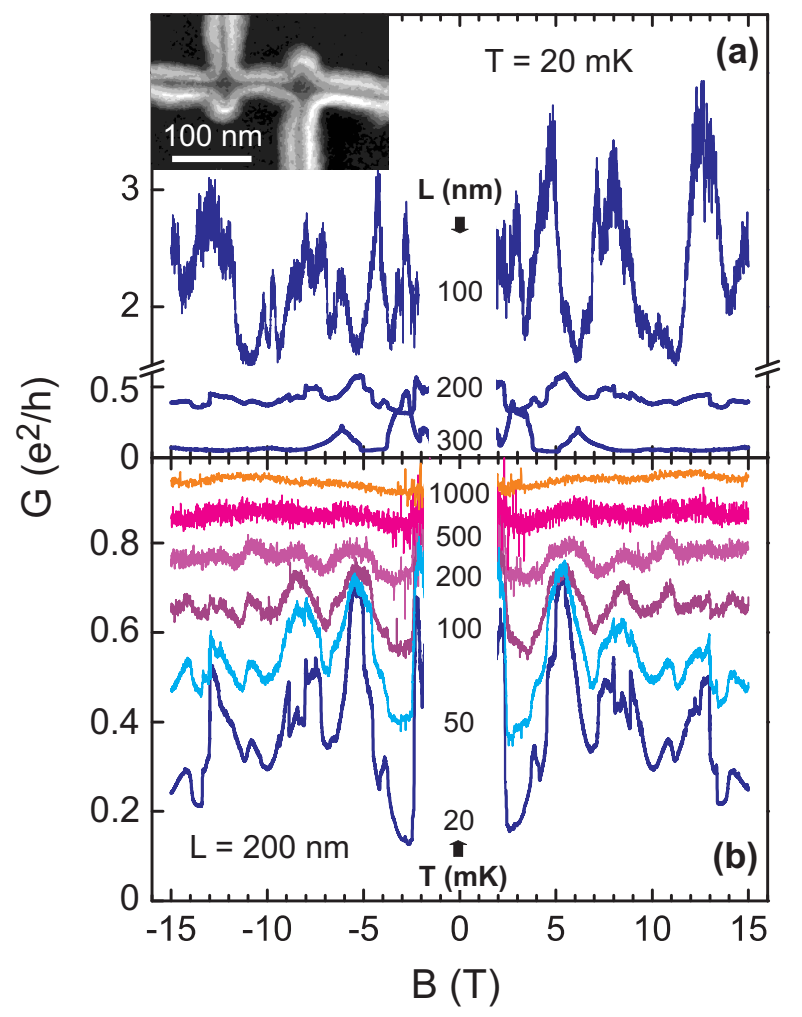

FIG. 2: (color online). (a) Conductance fluctuations for three wires of different length $L$. For the shortest wire the amplitude of the conductance fluctuations is about $e^{2} / h$, expected for conductors with all spatial dimensions smaller or comparable than $L_{\phi}$. The inset shows an electron micrograph of a 20 $\mathrm{nm}$ wide wire with a potential probe separation of $\sim 100 \mathrm{~nm}$ (the $100 \mathrm{~nm}$ wire). (b) $G$ vs. $B$ of the $200 \mathrm{~nm}$ wire for different temperatures between $20 \mathrm{mK}$ and $1 \mathrm{~K}$. In both experiments the current through the wire was $10 \mathrm{pA}$ (exception: $30 \mathrm{pA}$ at $1 \mathrm{~K})$. The conductance traces in the low $B$-regime have been omitted in both graphs.

than the longitudinal resistance $R_{x x}$, the conductance $G$ is simply obtained by inversion, $G=1 / R_{x x}$. The magnetic field dependence of $G$ of wires with $100 \mathrm{~nm}, 200 \mathrm{~nm}$ and $300 \mathrm{~nm}$ lengths, measured at $20 \mathrm{mK}$, is shown in Fig. 27. With decreasing lengths of the wires not only the conductance [30 but also the amplitude of the fluctuations increases. At $20 \mathrm{mK}$ and for the shortest wire of $100 \mathrm{~nm}$ length the amplitude of the fluctuations is of order $e^{2} / h$. The disappearance of the fluctuations for temperatures above $200 \mathrm{mK}$ is demonstrated for the $200 \mathrm{~nm}$ wire in Fig. 20 $\mathrm{b}$.

UCF result from correlations between different transmission paths through a disordered sample (21] and references therein). If the wire is smaller than $L_{\phi}$ in all three dimensions the fluctuation amplitude $\delta G=$ $\sqrt{\left\langle(G-\langle G\rangle)^{2}\right\rangle} \approx e^{2} / h$, where the bracket $\langle\ldots\rangle$ denotes averaging over $B$. Hence the data in Fig. 22, suggest that the phase coherence length is of order $100 \mathrm{~nm}$ for the lowest temperatures since $\delta G$ is of order $e^{2} / h$ for the $100 \mathrm{~nm}$ wire. Once the dimensions of the conductor exceed the 


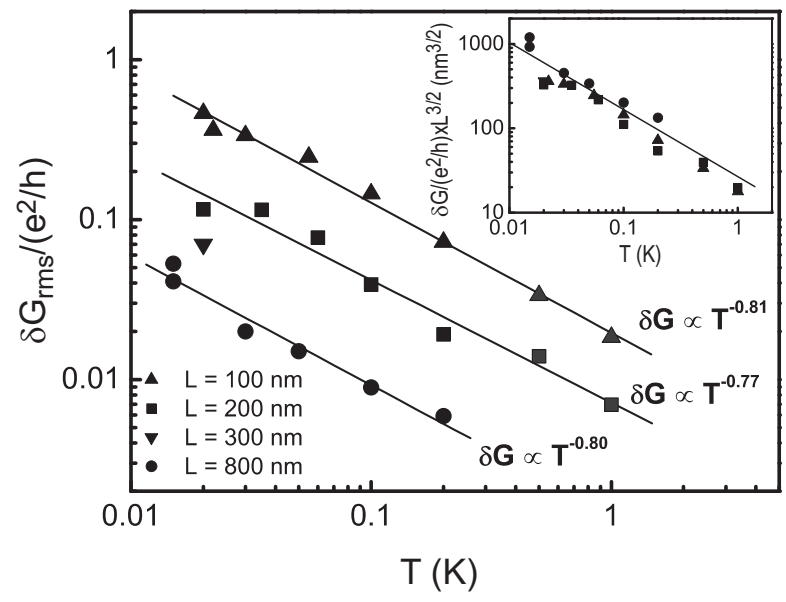

FIG. 3: $\delta G$ in units of $e^{2} / h$ for wires of length $100 \mathrm{~nm}$, $200 \mathrm{~nm}$ and $800 \mathrm{~nm}$. The measurements were carried out using currents of 10-500 pA. The maximum current at a given temperature was adjusted such that the fluctuation amplitude $\delta G$ was not affected. Inset: $\delta G L^{3 / 2} /\left(e^{2} / h\right)=L_{\phi}^{3 / 2}$ vs $T$.

phase coherence length $L_{\phi}=\sqrt{D \tau_{\phi}}$, with the coherence time $\tau_{\phi}, \delta G$ gets suppressed [20, 21]. To elucidate the conductance fluctuations further we study the fluctuation amplitude as a function of wire length and temperature. Corresponding data for wires with $L=100 \mathrm{~nm}, 200 \mathrm{~nm}$ and $800 \mathrm{~nm}$ are displayed in Fig. 3. The conductance amplitude $\delta G$ of all wires shows a power law dependence with an exponent close to $-3 / 4$. Carrying out the experiments at finite $T$ another length scale, the thermal length $L_{T}$ enters the stage. This length sets the scale over which thermal smearing causes dephasing. If the width $w$ and the thickness $t$ of the wires $t, w<L_{\phi}<L_{T}$ the dependence of $\delta G$ on $L_{\phi}$ is given by [20]

$$
\delta G=\frac{e^{2}}{h}\left[\frac{L_{\phi}}{L}\right]^{3 / 2}
$$

This formula is valid for one-dimensional (1D) conductors. Since we expect $L_{\phi}$ on the order of $100 \mathrm{~nm}$, width and thickness of our wires $(w \approx 20 \mathrm{~nm}, t \approx 50 \mathrm{~nm})$ are smaller than $L_{\phi}$ and the wires can be considered as 1Dconductors. Using the diffusion constant $D$ estimated above we obtain for $L_{T} \approx 150 \mathrm{~nm}$ at $15 \mathrm{mK}$. This means that the condition $t, w<L_{\phi}<L_{T}$ is realized in the (Ga,Mn)As wires, at least for low $T$. If the data in Fig. 3 are multiplied by $L^{3 / 2}$, all data points collapse on one line, shown in the inset of Fig. [3] Hence the scaling of $\delta G$ with $L$ is correctly described by Eq. (11). The temperature dependence of $L_{\phi}$ can then be directly read off the inset in Fig. 3 since, according to Eq. (11), the ordinate is given by $L_{\phi}^{3 / 2}$. Extrapolating to $10 \mathrm{mK}$ a phase coherence length of $\sim 100 \mathrm{~nm}$ is obtained.

The data in Fig. [3 imply that the temperature dependence of the phase coherence length is $\propto T^{-1 / 2}$ or, put in terms of the coherence time, $\tau_{\phi} \propto T^{-1}$. Here we have assumed that the $T$-dependence of $\delta G$ is, accor- dant with the data of Fig. 3, given by $T^{-3 / 4}$. We note that the $T$-dependence of $L_{\phi}$ remains unchanged even in the regime where $L_{T}<L_{\phi}<L$. There, instead of Eq. (11) $\delta G=\left(L_{T} / L\right)\left(L_{\phi} / L\right)^{1 / 2} e^{2} / h$ holds 20. Since $L_{T} \propto T^{-1 / 2}$ and $\delta G \propto T^{-3 / 4}$, again $L_{\phi} \propto T^{-1 / 2}$ results.

The $T$-dependence of $L_{\phi}$ contains information about the relevant dephasing mechanism and usually obeys a power law of the form $L_{\phi} \propto T^{-\alpha}$ where $\alpha$ depends on the dominating phase breaking mechanism and the dimensionality $d(d=1$ in our case). For $\alpha=-1 / 2$ no dephasing mechanism is readily available. Dephasing by electron-phonon scattering in 1D is usually described by $L_{\phi} \propto T^{-1}$ to $T^{-2}$ 22]. At low $T$ and reduced dimensions the small energy transfer Nyquist electron-electron interaction is a common source of dephasing [23]. The corresponding $L_{\phi} \propto T^{-1 / 3}$ dependence does, however, not describe our experimental result. A possible candidate for dephasing is critical electron-electron scattering described for strongly disordered metals in the vicinity of the MIT [24]. The corresponding inelastic scattering time was calculated to be $\propto T^{-1}$ but the calculations were done for bulk systems [25]. One should keep in mind, though, that in our system the charge is carried by holes. In addition the material is ferromagnetic. It is interesting to note here that - unlike for mesoscopic conductors containing magnetic impurities [26] - we do not observe saturation of coherence time at low temperatures.

As our analysis of UCFs suggests a phase coherence length of order $100 \mathrm{~nm}$ at mK-temperatures, the $\mathrm{AB}$ effect should be observable in sufficiently small (Ga,Mn)As rings. A corresponding experimental result is displayed in Fig. (4 an electron micrograph of the investigated ring is shown in Fig. 4a. From the micrograph we estimate an electrically active inner diameter $2 R_{i}$ of only $80 \mathrm{~nm}$, an outer diameter $2 R_{o}$ of $100 \mathrm{~nm}$. One half of the average ring perimeter is hence only slightly longer than $L_{\phi}$. The ring's conductance is compared to the conductance of a wire of $20 \mathrm{~nm}$ width and $200 \mathrm{~nm}$ length. From the ring's dimension we expect $\mathrm{AB}$ oscillations with a period $\Delta B$ between $h / e \pi R_{o}^{2}=0.53 \mathrm{~T}$ and $h / e \pi R_{i}^{2}=0.82 \mathrm{~T}$. Clear conductance oscillations in this range, absent for the wire geometry, are superimposed on the ring's conductance in Fig. 4b. A bar of $\Delta B=0.7 \mathrm{~T}$ length is displayed as a guide for the eye there. A Fourier transform of both, ring

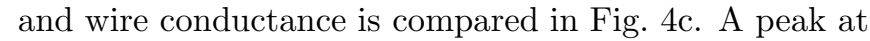
$1 / \Delta B \sim 1.4 \mathrm{~T}^{-1}$ appears in the Fourier transform lying within the window expected for $\mathrm{AB}$ oscillations. While clear $\mathrm{AB}$ oscillation are observable in our smallest ring, the analysis is complicated by two factors: (i) superimposed upon the oscillations are conductance fluctuations with a similar magnetic field scale as the corresponding areas enclosed by trajectories are similar [27]. Hence fluctuations and oscillations are hard to separate. (ii) The finite width of the ring causes an uncertainty in the expected $\Delta B$ of the oscillations. Trajectories traveling along the outer side of the ring might - in contrast to paths along the inner perimeter - contribute less to the 
(a)

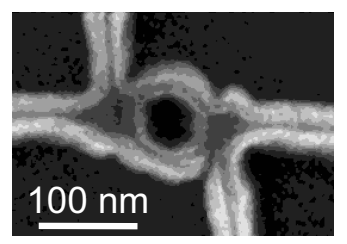

(c)

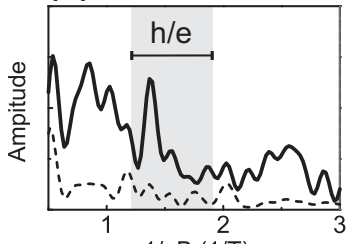

(b)

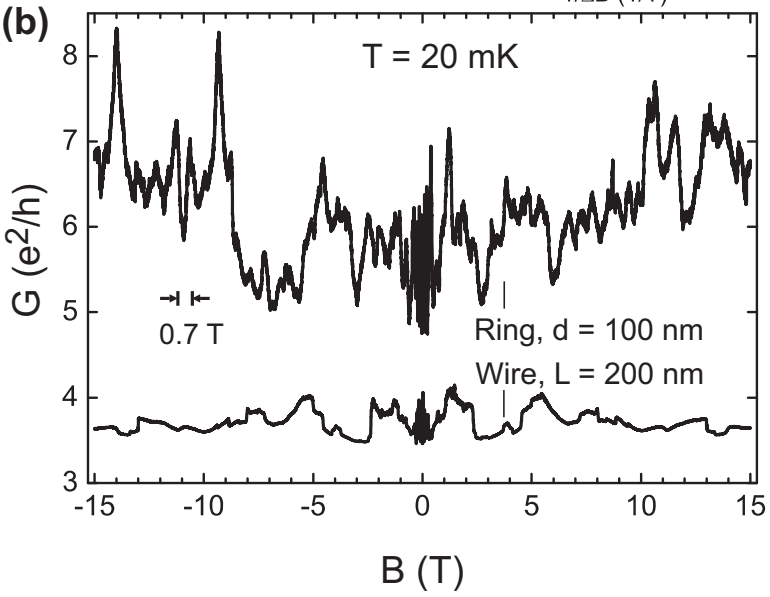

FIG. 4: (a) Electron micrograph of a (Ga,Mn)As ring sample with a diameter of $\sim 100 \mathrm{~nm}$. (b) Comparison of the magnetoconductance trace of the ring sample with the conductance of a wire of comparable length and $20 \mathrm{~nm}$ width. (c) Corresponding FFT taken from the conductance of ring and wire. The region where $\mathrm{AB}$ oscillations are expected is high-lighted.
$\mathrm{AB}$ effect.

By resolving both universal conductance fluctuations and Aharonov-Bohm effect we have shown clear phase coherent effects in ferromagnetic (Ga,Mn)As nanostructures. The phase coherence length in the material investigated was $\sim 100 \mathrm{~nm}$ at $10 \mathrm{mK}$ and showed a $T^{-1 / 2}$ temperature dependence. This means that the relevant coherence time follows a $T^{-1}$ law.

Acknowledgements

We thank T. Dietl, J. Fabian, and C. Strunk for valuable discussions and the German Science Foundation (DFG) for their support via the Research Unit FOR370 and the Collaborative Research Grant SFB689.
[1] H. Ohno, H. Munekata, T. Penney, S. von Molnár, and L. L. Chang, Phys. Rev. Lett. 68, 2664 (1992).

[2] H. Ohno, A. Shen, F. Matsukura, A. Oiwa, A. Endo, S. Katsumoto, and Y. Iye, Appl. Phys. Lett. 69, 363 (1996).

[3] H. Ohno, Science 281, 951 (1998).

[4] H. Ohno, J. Magn. Magn. Mater. 200, 110 (1999).

[5] H. Ohno and F. Matsukura, Solid State Commun. 117, 179 (2001).

[6] T. Dietl, Semicond. Sci. Technol. 17, 377 (2002).

[7] A. H. MacDonald, P. Schiffer, and N. Samarth, Nature Materials 4, 195 (2005).

[8] H. Ohno, D. Chiba, F. Matsukura, T. Omiya, E. Abe, T. Dietl, Y. Ohno, and K. Ohtani, Nature 408, 944 (2000).

[9] A. Oiwa, S. Katsumoto, A. Endo, M. Hirasawa, Y. Iye, H. Ohno, F. Matsukura, A. Shen, and Y. Sugawara, Solid State Commun. 103, 209 (1997).

[10] T. Dietl, A. Haury, and Y. M. d'Aubigné, Phys. Rev. B 55, R3347 (1997).

[11] T. Jungwirth, K. Y. Wang, J. Maek, K. W. Edmonds, J. König, J. Sinova, Polini, N. A. Goncharuk, A. H. MacDonald, M. Sawicki, et al., Phys. Rev. B 72, 165204 (2005).

[12] K. W. Edmonds, P. Bogusawski, K. Y. Wang, R. P. Campion, S. N. Novikov, N. R. S. Farley, B. L. Gallagher, C. T. Foxon, M. Sawicki, T. Dietl, et al., Phys. Rev.
Lett. 92, 037201 (2004).

[13] K. Y. Wang, R. P. Campion, K. W. Edmonds, M. Sawicki, T. Dietl, C. T. Foxon, and B. L. Gallagher, AIP Conf. Proc. 772, 333 (2005).

[14] A. M. Nazmul, T. Amemiya, Y. Shuto, S. Sugahara, and M. Tanaka, Phys. Rev. Lett. 95, 017201 (2005).

[15] M. Reinwald, U. Wurstbauer, M. Döppe, W. Kipferl, K. Wagenhuber, H.-P. Tranitz, D. Weiss, and W. Wegscheider, J. Crystal Growth 278, 690 (2005).

[16] T. Dietl, H. Ohno, and F. Matsukura, Phys. Rev. B 63, 195205 (2001).

[17] D. V. Baxter, D. Ruzmetov, J. Scherschligt, Y. Sasaki, X. Liu, J. K. Furdyna, and C. H. Mielke, Phys. Rev. B 65, 212407 (2002).

[18] E. L. Nagaev, Phys. Rev. B 58, 816 (1998).

[19] F. Matsukura, M. Sawicki, T. Dietl, D. Chiba, and H. Ohno, Physica E 21, 1032 (2004).

[20] P. A. Lee, A. D. Stone, and H. Fukuyama, Phys. Rev. B 35, 1039 (1987).

[21] S. Washburn and R. A. Webb, Rep. Prog. Phys. 55, 1311 (1992).

[22] J. J. Lin and J. P. Bird, J. Phys.: Condens. Matter 14, R501 (2002).

[23] B. L. Altshuler, A. G. Aronov, and D. E. Khmelnitsky, J. Phys. C. 15, 7367 (1982).

[24] P. Dai, Y. Zhang, and M. P. Sarachik, Phys. Rev. B 46, 6724 (1992). 
[25] D. Belitz and K. I. Wysokinski, Phys. Rev. B 36, R9333 (1987).

[26] F. Pierre, A. B. Gougam, A. Anthore, H. Pothier, D. Esteve, and N. O. Birge, Phys. Rev. B 68, 085413 (2003).

[27] S. Washburn and R. Webb, Adv. Phys. 35, 375 (1986).

[28] T. Omiya, F. Matsukura, T. Dietl, Y. Ohno, T. Sakon, M. Motokawa, and H. Ohno, Physica E 7, 976 (2000).

[29] We obtained $p$ by probing the best fit to $R_{x y}=R_{0} B / t+$ $R_{s} M / t$ [28] and extracting $p$ from the ordinary Hall constant $R_{0}=1 /$ pe above $B>0.2 \mathrm{~T}$ where the magnetization $M$ is saturated. Here the magnetic field $B$ is perpen- dicular to the sample's plane, $t$ is the layer thickness, and $R_{s}$ is the anomalous Hall term. $R_{s}$ depends on $R_{\text {sheet }}$ as $R_{s} \propto R_{\text {sheet }}$ or $R_{s} \propto R_{\text {sheet }}^{2}$. Our fits showed clearly a $R_{s} \propto R_{\text {sheet }}$ dependence for the material investigated.

[30] We have checked that we are still in a regime where the conductance of the wires scales as $1 / L$ whith $L$ the length of a wire. Sample to sample fluctuations of the actual width in our extra narrow wires cause deviations from this scaling if, as in Fig. 27, different samples with the same nominal width are compared. 